\title{
EFEITOS DE UM PROGRAMA DE CAMINHADA SUPERVISIONADA NA QUALIDADE DE VIDA DE INDIVÍDUOS COM SÍNDROME METABÓLICA
}

\section{EFFECTS OF A SUPERVISED WALKING PROGRAM ON THE QUALITY OF LIFE OF SUBJECTS WITH METABOLIC SYNDROME}

\author{
Tadeu Candido da Silva Santos ${ }^{1}$ \\ Matheus Guedes Fernandes Silva ${ }^{2}$ \\ Tatiane Lopes Patrocínio da Silva ${ }^{3}$
}

RESUMO: A realização de exercício físico regular é a terapia não-medicamentosa de primeira escolha para tratamento de indivíduos com Síndrome Metabólica (SM), com redução de peso, melhora da sensibilidade à insulina, diminuição dos níveis de glicose, prevenção do aparecimento de diabetes e melhora da qualidade de vida (QV). O objetivo deste estudo foi avaliar os benefícios de um programa de caminhada supervisionada (PCS) na QV de indivíduos com SM. Participaram 8 voluntários com SM (63 $\pm 9,65$ anos). Antes do PCS todos realizaram o teste de caminhada de seis minutos (TC6'), o teste de caminhada com carga progressiva ou Shuttle Walk Test (TCP) e foi aplicado o questionário de QV SF-36, verificando as variáveis: distância percorrida (DP), percepção de esforço pela escala de Borg, pressão arterial e frequência cardíaca em repouso e ao final dos testes. Os voluntários foram orientados a caminhar 30 minutos, 2 vezes por semana por 10 semanas, sempre com a supervisão do pesquisador, e, ao final do PCS, foram reavaliados com o mesmo protocolo da avaliação. Após a verificação da normalidade dos dados, optou-se pelo teste Wilcoxon para variáveis dependentes e para análise de correlação o teste de Speerman. No TC6' e no TCP, observou-se aumento na DP com menor Borg final. Na avaliação da QV observou-se aumento na pontuação total do SF-36, bem como nos domínios analisados de forma individualizada. Conforme os resultados obtidos, conclui-se que o PCS aumentou a tolerância aos esforços, com menor sensação de dispneia e melhora da QV em indivíduos com SM.

Palavras-chave: Síndrome Metabólica; qualidade de vida; atividade física.

ABSTRACT: Regular exercise is the first choice of a non-drug therapy to treat patients with metabolic syndrome, because it reduces weight, improves insulin sensitivity, decreases glucose levels, prevents the onset of diabetes, and improves the quality of life (QL). The aim of this study was to evaluate the benefits of a supervised walking program (SWP) on the QL of individuals with metabolic syndrome. Eight volunteers with metabolic syndrome (63 \pm 9.65 years) participated in the study. Before the SWP, all of them did a six-minute walking test (6MWT), a Shuttle Walk Test (SWT), and filled out the SF-36 questionnaire to assess QL. The variables assessed included: distance walked (DW), perceived exertion using the Borg scale, blood pressure, as well as heart rate at rest and after the tests. The volunteers were instructed to walk 30 minutes, 2 times per week, for 10 weeks, always with the supervision of the researcher, and at the end of the SWP were reevaluated with the same assessment protocol. After verifying the normality of the data, we chose the Wilcoxon test for dependent variables and the Speerman test for correlation analysis. In the 6MWT and the SWT, there was an increase in DW with lower Borg at the end of test. QL evaluation showed an increase in the total score of the SF-36 and improvements in the areas analyzed individually. These results indicate that the SWP increased tolerance to effort, with less dyspnea and improved QL in individuals with metabolic syndrome.

Keywords: Metabolic Syndrome; quality of life; physical activity.

\footnotetext{
${ }^{1}$ Graduado em Fisioterapia - UNITAU.

E-mail: tad_silva@ig.com.br

${ }^{2}$ Especialista em Fisiologia do Exercício - UNIFESP.

E-mail: matheusgfs@bol.com.br

${ }^{3}$ Mestre em Fisioterapia - UFSCar e Docente da UNITAU.

E-mail: tati.patrocinio@gmail.com
} 


\section{INTRODUÇÃO}

Nos últimos anos, a Síndrome Metabólica (SM) tem sido motivo de grande interesse e debate na literatura médica uma vez que agrupa uma série de fatores de risco para doenças cardiovasculares (MANNA; DAMIANI; SETIAN, 2006) como morte súbita, infarto agudo do miocárdio e tem-se tornado, atualmente, um dos maiores desafios em termos de saúde pública mundial (RIGO et al., 2009).

Indivíduos portadores de SM apresentam uma probabilidade real de aumento na taxa futura de morbidade e mortalidade de origem cardiovascular, com grande impacto sócio-econômico não só para - Brasil, mas também para todos os países em desenvolvimento (MOTA et al., 2006).

Junqueira, Romeu Filho e Junqueira (2009) consideram a importância do controle dos fatores de risco e estratificação deles no que tange à associação entre HAS, DM2 ou $\mathrm{RI}$, dislipidemia e obesidade visceral e torna-se necessário um maior empenho em delinear fatores prognósticos na prevenção e progressão da doença cardiovascular.

Dentre as doenças crônicas, a HAS e DM2 exigem alterações de comportamento em relação à dieta, ingestão de medicamentos e o estilo de vida. $O$ tratamento inadequado ou 0 não reconhecimento da importância das complicações que decorrem destas patologias podem comprometer a qualidade de vida (QV) da população (MIRANZI et al., 2008).

Estratégias de modificação do estilo de vida sustentável são necessárias para tratar a obesidade e reduzir fatores de risco cardiovascular em pessoas com SM, de forma intensiva, individualizada, baseadas em dieta e AF semanal de forma aeróbia, como caminhada, e alongamentos com monitoração da frequência cardíaca ( $F C)$. $O$ treinamento ativo deve incluir $\mathrm{o}$ apoio social e acompanhamento clínico a longo prazo para mudar o estilo de vida, melhorar a composição corporal, diminuir os prejuízos cardiometabólicos e favorecer a QV (PETTMAN et al., 2008).

A QV possui vinculações importantes com a aptidão física e estas se manifestam mais visivelmente quando analisados os efeitos benéficos da AF sistemática para o bem-estar das pessoas. A adoção de comportamentos saudáveis evidencia importante papel coadjuvante do exercício e da AF constante na busca de um estilo de vida positivo como forma de prevenção de problemas de saúde, além de interferir no processo saúde-doença e favorecer a QV (CAMPOS; PORTO, 2009).

A QV é tema de grande importância, principalmente por envolver aspectos inerentes ao indivíduo e considerar a percepção da pessoa sobre o seu estado de saúde em grandes domínios ou dimensões de sua vida. Na área da saúde, em especial à cardiologia, a análise da QV deve ser considerada em pesquisas com seres humanos, e não somente dados estatísticos específicos da efetividade de uma técnica ou intervenção, em particular (WINKELMANN; MANFROI, 2008).

\section{FUNDAMENTAÇÃO TEÓRICA}

\subsection{Definição de Síndrome Metabólica}

A SM é um transtorno complexo, com 
múltiplos fatores de risco cardiovascular e corresponde à clássica definição de uma síndrome, com um conjunto de sinais e sintomas associado a uma base fisiopatológica comum: a adiposopatia centrípeta (deposição central de gordura) e RI (McLELLAN et al., 2007), índices antropométricos, dislipidemias, HAS, alterações do metabolismo de carboidratos, estados pró-trombóticos e pró-inflamatórios (MANNA; DAMIANI; SETIAN, 2006) que levam a um aumento da mortalidade geral, especialmente de origem circulatória (JUNQUEIRA; ROMEO FILHO; JUNQUEIRA, 2009).

Esses fatores de risco evidenciam que a SM provem de um metabolismo anormal, com um aumento para o desenvolvimento de doença cardiovascular aterosclerótica (DCVA) e DM2. Pacientes com SM têm de 1,5 a 3 vezes mais risco para DCVA e 5 vezes mais risco para o DM2 (GELONESE, 2006).

Carvalho e Rabelo

(2009) evidenciaram que, independente do grupo ou entidade que defina a SM, os fatores de risco permaneceram os mesmos: obesidade (especialmente a abdominal), níveis pressóricos elevados, distúrbios no metabolismo da glicose e hipertrigliceridemia e/ou baixos níveis de HDL colesterol.

As doenças cardiovasculares representam a principal causa de morte no mundo. A SM é hoje um dos maiores desafios para a saúde pública mundial, por associar-se aos fatores de risco cardiovascular e DM2 e diversos critérios diagnósticos têm como objetivo identificar precocemente os indivíduos portadores da síndrome (LUNA, 2007).

Atualmente, existem diferentes consensos para o diagnóstico da SM (Quadro 1), como o Third Report of the National Cholesterol Education Program Expert Panel on Detection, Evaluation, and Treatment of High Blood Cholesterol in Adults - Panel III (NCEP-ATP III), a Federação Internacional de Diabetes (IDF) e a OMS (NAKAZONE et al., 2007; COELHO et al., 2007). Embora existam diversas controvérsias a respeito dos critérios diagnósticos, o IDF estima que pelo menos um quarto da população mundial tem a síndrome (MIRANZI et al., 2008).

\section{Quadro 1 - Consensos para diagnóstico da Síndrome Metabólica}

\begin{tabular}{|c|c|c|}
\hline IDF (2005) & NCEP (2001) & OMS (2005) \\
\hline $\begin{array}{l}\text { Diagnóstico se alteração de } \\
\text { glicemia e mais dois critérios }\end{array}$ & $\begin{array}{l}\text { Diagnóstico se três dos cinco } \\
\text { critérios presentes }\end{array}$ & $\begin{array}{l}\text { Diagnóstico se alteração de } \\
\text { glicemia e mais dois critérios }\end{array}$ \\
\hline $\begin{array}{c}\text { Glicemia de jejum } 100-125 \mathrm{mg} / \mathrm{dL} \\
\text { ou DM2 }\end{array}$ & Glicemia $110-125$ mg/dL & $\begin{array}{l}\text { Intolerância à glicose, } \\
\text { DM2 ou RI }\end{array}$ \\
\hline $\begin{array}{l}\text { Homens: } C A \geq 94 \mathrm{~cm} \\
\text { Mulheres: } C A \geq 80 \mathrm{~cm}\end{array}$ & $\begin{array}{l}\text { Homens: } \mathrm{CA}>102 \mathrm{~cm} \\
\text { Mulheres: } \mathrm{CA}>88 \mathrm{~cm}\end{array}$ & $\begin{array}{c}\text { IMC > } 30 \text { e } R C Q>0,9 \text { para Homens } \\
\text { e }>0,85 \text { para Mulheres }\end{array}$ \\
\hline $\begin{array}{c}\text { TG } \geq 150 \mathrm{mg} / \mathrm{dL} \\
\text { ou } \mathrm{HDL}<40 \text { para Homens } \\
\text { e }<50 \mathrm{M} \text { para Mulheres }\end{array}$ & $\begin{array}{l}\text { TG } \geq 150 \mathrm{mg} / \mathrm{dL} \\
\text { ou } \mathrm{HDL}<40 \text { para Homens } \\
\text { e }<50 \text { M para Mulheres }\end{array}$ & $\begin{array}{c}\mathrm{TG} \geq 150 \mathrm{mg} / \mathrm{dL} \\
\text { ou } \mathrm{HDL}<35 \mathrm{H} \text { para Homens } \\
\mathrm{e}<39 \text { para Mulheres }\end{array}$ \\
\hline $\begin{array}{c}\text { HAS em tratamento } \\
\text { ou } \mathrm{PA} \geq 130 \times 85 \mathrm{mmHg} \\
\end{array}$ & $P A \geq 130 \times 85 \mathrm{mmHg}$ & $\begin{array}{c}\text { HAS em tratamento ou } \mathrm{PA} \geq 160 \times 90 \mathrm{mmHg} \\
\text { Microalbuminúria } \geq 20 \mathrm{mcg} / \mathrm{min}\end{array}$ \\
\hline $\begin{array}{l}\text { IDF = International Diabetes } \\
\text { Education Program; OMS = O } \\
\text { HDL = Lipoproteinas de Alta } \\
\text { IMC = Índice de Massa Corpóre }\end{array}$ & $\begin{array}{l}\text { Ion (Federação Internaciona } \\
\text { lção Mundial de Saúde; CA } \\
\text { lade; HAS = Hipertensão Ar } \\
=\text { Relação Cintura: Quadril }\end{array}$ & $\begin{array}{l}\text { etes); NCEP = US National Cholesterol } \\
\text { erência Abdominal; TG = Triglicerídeos; } \\
\text { têmica; DM2 = diabetes mellitus tipo2; } \\
\text { essão Arterial; RI = Resistência à Insulina. }\end{array}$ \\
\hline
\end{tabular}

Fonte: Adaptação de Lottenberg S. A. et al., 2007. 
O NCEP-ATP III, desde 2001, estabelece como diagnóstico a presença de pelo menos três dentre cinco critérios (CARVALHO; RABELO, 2009) como CA elevada (> $102 \mathrm{~cm}$ para os homens e > 88 $\mathrm{cm}$ para mulheres), TG elevados (> 150 $\mathrm{mg} / \mathrm{dl})$, redução $\mathrm{HDL}(<40 \mathrm{mg} / \mathrm{dL}$ para homens, $<50 \mathrm{mg} / \mathrm{dl}$ para mulheres), alta taxa de glicemia em jejum (> 110-125 mg/dL) e HAS (> 130/85 mmHg) (LOTTENBERG; GLEZER; TURATTI, 2007; NAKAZONE et al., 2007; TSAl et al., 2007).

O IDF, em 2005, associou a obesidade abdominal com dois ou mais componentes para o diagnóstico e definiu parâmetros de acordo com o grupo racial do indivíduo (NAKAZONE et al., 2007), através da CA e adaptou ao perfil antropométrico das populações dos diferentes continentes (MANNA; DAMIANI; SETIAN, 2006) o que possibilitou que o diagnóstico pudesse ser usado e padronizado em qualquer parte do mundo e adequado para cada etnia (BARBOSA et al., 2010).

Em 2005, foi publicada a I Diretriz Brasileira de Diagnóstico e Tratamento da Síndrome Metabólica, com o apoio da Sociedade Brasileira de Cardiologia, que utilizou o critério NCEP-ATP III (BARBOSA et al., 2010).

De acordo com a I Diretriz, a SM é um transtorno complexo, de importância epidemiológica, relacionada à deposição central de gordura e a $\mathrm{Rl}$, responsável pelo aumento da mortalidade cardiovascular estimada em 2,5 vezes. O risco é aumentando pela alta prevalência da tríade lipídica (hipertrigliceridemia, níveis baixos de HDL e níveis altos de partículas pequenas e densas de LDL).
Estima-se que, em diferentes populações adultas, há uma elevada prevalência, que varia entre $25 \%$ e $35 \%$, sendo mais frequente em mulheres (COELHO et al., 2007). A síndrome aumenta com a idade, em homens e mulheres, alcançando $50 \%$ entre 60 e 69 anos (BARBOSA et al., 2010). Não foram encontrados estudos sobre a prevalência da SM com dados representativos da população brasileira. No entanto, estudos em diferentes populações, como a mexicana, a norte-americana e a asiática, revelam prevalências elevadas da SM, dependendo do critério utilizado e das características da população estudada, variando as taxas de $12,4 \%$ a $28,5 \mathrm{em}$ homens e de $10,7 \%$ a $40,5 \%$ em mulheres.

\subsection{Qualidade de Vida e Síndrome Metabólica}

Segundo a OMS, QV é a percepção do indivíduo de sua posição na vida, no contexto da cultura e do sistema de valores nos quais vive, considerando objetivos, expectativas, padrões e preocupação. Esta definição enfatiza a valorização subjetiva, em um contexto cultural, social e ambiental, onde estão presentes seis domínios: físico, psicológico, nível de interdependência, relacionamento social, ambiente e comportamentos pessoais (SILVA; JESUS; SANTOS, 2007).

A QV apresenta vinculações importantes com a aptidão física e estas se manifestam mais claramente quando considerados os efeitos benéficos da $\mathrm{AF}$ para o bem-estar do indivíduo. A estreita associação entre a prática regular de AF e a 
prevenção de problemas de saúde interfere no processo saúde-doença e propicia melhora na QV (CAMPOS; PORTO, 2009).

Dentre os instrumentos de medida da QV, o SF-36 (MOS SF-36-Medical Outcomes Study, Short Form - 36, Health Survey) avalia o perfil de saúde, é de fácil administração, compreensão e amplamente utilizado na literatura (CAVALCANTE et al., 2007).

Em um estudo norte-americano, Sullivan et al. (2007) evidenciaram que indivíduos com alterações cardiometabólicas como sobrepeso, obesidade, DM2, HAS e hiperlipidemia apresentaram um impacto significativo e negativo na QV na população dos Estados Unidos.

Tsai et al. (2007) estudaram a associação entre SM e QV e constataram que houve menor pontuação na função física e sub-escalas de saúde geral do questionário SF-36. Indivíduos com SM apresentaram menor QV, sintomas de depressão, uso de álcool ou tabaco e estresse, bem como um significativo aumento do IMC quando comparado aos indivíduos sem a síndrome.

Em um estudo sobre a QV em indivíduos hipertensos em tratamento ambulatorial apenas por meio de medicação anti-hipertensiva, Cavalcante et al. (2007) revelaram que não houve melhora em nenhum domínio do SF-36.

Miranzi et al. (2008), em um estudo da QV em indivíduos portadores de DM2 e HAS, tanto na prevenção, promoção e recuperação da saúde, acompanhados por uma equipe de Saúde da Família revelaram que o programa foi tido como positivo entre os entrevistados na maioria dos domínios mensurados: relações sociais, estado físico, psicológico e meio ambiente.

Em outro estudo, Pinotti, Mantovani e Giacomozzi (2008) avaliaram a QV em indivíduos hipertensos e verificaram que a HAS interferiu de maneira negativa, principalmente em relação ao trabalho, hábitos de vida e autocuidado, decorrente do caráter crônico da doença.

Brito et al. (2008), em um estudo com hipertensos de uma Unidade Básica de Saúde com a utilização do SF-36, identificaram comprometimento em todos os domínios do questionário, o que sugere um comprometimento global de suas vidas ao longo dos anos com a presença da doença.

Han et al. (2009) avaliaram a QV através de dois questionários, o EuroQol e o KOQOL, e demonstraram que pessoas com SM apresentaram uma QV significativa menor em comparação àquelas sem a doença e que são mais propensas a apresentarem problemas com a saúde física, saúde relacionada ao trabalho, vida cotidiana, vida sexual, angústia com a dieta e dor ou desconforto.

\subsection{Síndrome metabólica e atividade física}

A realização de exercício físico regular é considerada a terapia não-medicamentosa de primeira escolha para o tratamento de pacientes com SM, com redução expressiva da CA e diminuição da gordura visceral, melhora da sensibilidade à insulina, diminuição dos níveis plasmáticos de glicose e prevenção do aparecimento de DM2 (ELIAS et al., 2008). A incidência de diabetes diminui em $6 \%$ com cada aumento de $500 \mathrm{kcal} / \mathrm{semana}$ de gasto energético 
com o treinamento físico (SATO et al., 2007).

O exercício promove redução da gordura corporal e envolve o aumento do GET, sem um correspondente aumento no consumo de energia. A longo prazo, a AF com intensidade, duração e frequência suficientes tem um efeito favorável na redução do peso e distribuição da gordura corporal. A eficácia do exercício para induzir a perda de peso está diretamente relacionada ao grau inicial da obesidade e do gasto total de energia (PITSAVOS et al., 2006).

A realização de AF regular, de acordo com os efeitos endocrinológicos e metabólicos, melhora a utilização de glicose e dos AGL no músculo e reduz os níveis de glicose no sangue em diabéticos (SATO et al., 2007). Pugliese et al. (2009), em seus estudos, verificaram que 0 exercício aumenta 0 fluxo sanguíneo regional, recrutamento e permeabilidade capilar, com consequente aumento do transporte de glicose pela membrana e entrega ao músculo, o que diminuem os fatores de risco cardiovascular modificáveis, incluindo o controle glicêmico.

O treinamento físico está associado ainda com alterações nos marcadores do metabolismo do colesterol, com aumento da absorção, bem como regulação da produção. Ocorre melhora do perfil lipoprotéico, com redução dos níveis de LDL- colesterol e aumento dos níveis de HDL-colesterol (WILUND et al., 2009), diminuição nos níveis plasmáticos de TG e melhoria da HAS leve, exercendo um efeito inibidor sobre 0 desenvolvimento e progressão da arterosclerose (SATO et al., 2007). Longos períodos de treinamento promovem melhora dos níveis pressóricos arteriais em indivíduos portadores de diabetes tipo II e síndrome metabólica (BALDUCCl et al., 2009).

\section{MÉTODOS}

Este estudo foi realizado no período de maio a novembro de 2010, na Clínica de Fisioterapia da Universidade de Taubaté, Taubaté (SP), Brasil, na qual foram avaliados 8 voluntários com SM, 1 do sexo masculino e 7 do sexo feminino, com idade entre 53 e 82 anos (média de $63 \pm 9,65$ anos), selecionados através da aplicação do questionário de seleção da amostra.

Todos os voluntários assinaram um termo de consentimento, livre e esclarecido. O estudo foi previamente aprovado pelo Comitê de Ética em Pesquisa da Universidade de Taubaté sob $n^{\circ} 199 / 10$, em reunião de 11 de junho de 2010, segundo a normatização do Conselho Nacional de Saúde do Ministério da Saúde n 196/69, para pesquisa em humanos.

Os critérios de inclusão levaram em consideração a classificação de SM de acordo com o NCEP (2001) Glicemia 110$125 \mathrm{mg} / \mathrm{dL}$, Circunferência abdominal em homens $>102 \mathrm{~cm}$ e mulheres $>88 \mathrm{~cm}$, nível de triglicerídeo $\geq 150 \mathrm{mg} / \mathrm{dL}$, ou $\mathrm{HDL}<40$ para Homens e $<50 \mathrm{M}$ para Mulheres e PA $\geq 130 \times 85 \mathrm{mmHg}$. Os pacientes que apresentaram pelo menos três dos cinco critérios adotados foram incluídos no estudo.

Os critérios de exclusão para a participação deste estudo foram: presença de problemas ortopédicos, músculos-esqueléticos ou neuromusculares; problemas cardíacos não controlados; e 
realização de atividade física regular.

\subsection{Procedimento Experimental}

O protocolo experimental de avaliação foi composto da realização de anamnese, aplicação do questionário de QV SF-36, coleta dos sinais vitais, com medidas da Pressão Arterial (PA) e FC em repouso e exame físico geral, aplicação do Teste de Caminhada de 6 minutos (TC6), com intervalo de descanso de trinta minutos e aplicação do Teste de Caminhada de Carga Progressiva (TCP), em dias e horas marcadas com prévia instrução de como seriam realizados.

Os voluntários foram orientados a usarem roupas leves e calçados apropriados, realizar uma refeição leve e não realizar exercício vigoroso duas horas antes do início da avaliação. O Programa de Caminhada Supervisionada (PCS) teve uma duração de 10 semanas, duas vezes por semana em dias não consecutivos, com um total de 20 sessões de aproximadamente uma hora; a assiduidade dos voluntários foi garantida com reposição da sessão em outro dia da semana sempre que o voluntário se ausentasse da sessão de treinamento. Ao término do PCS, os voluntários foram submetidos à reavaliação, com o mesmo protocolo da avaliação. Durante o PCS os pesquisadores estavam presentes orientando os voluntários e foi utilizado o seguinte protocolo:

Após a chegada do voluntário ao ambulatório de reabilitação cardíaca, ele era orientado a permanecer sentado, e, após dez minutos de repouso, era realizada a aferição da pressão arterial do voluntário. a) Início: aproximadamente 2 minutos para mensuração da FC de repouso;

b) Aquecimento: aproximadamente 10 minutos para realização de exercícios de membros superiores e inferiores associados à mobilidade de tronco, exercícios dinâmicos e alongamentos gerais;

c) Treinamento físico: 30 minutos de caminhada em ambiente externo e plano na velocidade determinada pela FC, de 50 a $60 \%$ da FC máxima estimada;

d) Desaquecimento:

aproximadamente 5 minutos com alongamentos gerais.

\subsection{Análise Estatística}

Após verificação da normalidade dos dados, optou-se por testes não paramétricos. Para análise estatística dos dados utilizou-se o teste Wilcoxon para variáveis dependentes, na comparação antes e após o PCS, e para análise de correlação utilizou-se o teste de speerman. O pacote estatístico utilizado foi o Statsoft STATISTICA 6.0 e o nível de significância adotado foi de $p \leq 0,05$.

\section{RESULTADOS}

De acordo com os resultados do TC6' antes e após o PCS, com relação à variável DP, foram encontrados aumento dos escores de $434,88 \mathrm{~m}$ para $559,88 \mathrm{~m}$. O mesmo pode ser observado na avaliação e reavaliação dos voluntários no TCP com aumento de $180,25 \mathrm{~m}$ para $230,13 \mathrm{~m}$.

Com relação à variável Borg, tanto no TC6' e no TCP, houve diminuição da 
sensação subjetiva de esforço avaliada através da escala de Borg, modificada no final dos testes. No TC6' a sensação de dispnéia passou de 3,5 para 1,5 e de 3,4 para 2 no TCP.
Conforme demonstrado na Tabela 1, através da comparação antes e após o PCS obtidas pela aplicação do TC6', houve relevância significativa nas variáveis DP $(p=0,0117)$, FC final $(p=0,0117)$ e Borg final $(p=0,0117)$.

Tabela 1 - Comparação das variáveis DP, FC inicial e final, Borg inicial e final e PAs e PAd inicial e final obtidas no TC6' antes e após o PCS com resultados apresentados em médias e desvio padrão e com os resultados do teste de Wilcoxon para variáveis dependentes.

\begin{tabular}{lccc}
\hline Variáveis & $\begin{array}{c}\text { Programa de Caminhada Supervisionada } \\
\text { Avaliação }\end{array}$ & $\begin{array}{c}\text { Resultado } \\
\text { Rstatístico } \\
\mathbf{P}\end{array}$ \\
\hline RP & $434,88 \pm 39,01$ & $559,88 \pm 24,29$ & 0,0117 \\
FC inicial & $76,50 \pm 13,83$ & $73,88 \pm 10,92$ & 0,4838 \\
FC final & $132,38 \pm 35,17$ & $99,00 \pm 11,46$ & 0,0117 \\
Borg Inicial & $0,00 \pm 0,00$ & $0,00 \pm 0,00$ & 1,0000 \\
Borg Final & $3,50 \pm 0,76$ & $1,50 \pm 0,53$ & 0,0117 \\
PAs Inicial & $133,75 \pm 11,88$ & $133,75 \pm 9,16$ & 1,0000 \\
PAs Final & $152,50 \pm 14,88$ & $145,00 \pm 10,69$ & 0,0679 \\
PAd Inicial & $85,00 \pm 10,69$ & $80,00 \pm 5,35$ & 0,2013 \\
PAd Final & $95,00 \pm 5,35$ & $90,00 \pm 7,56$ & 0,1775 \\
\hline
\end{tabular}

$\mathrm{DP}=$ distância percorrida $(\mathrm{m}) ; \mathrm{FC}=$ Frequência cardíaca $(\mathrm{bpm})$; Borg = pontuação na escala de Borg; PAs = Pressão Arterial sistólica $(\mathrm{mmHg}) ; \mathrm{PAd}=$ Pressão Arterial diastólica $(\mathrm{mmHg}) ; \mathrm{p} \leq 0,05$. * Significativo para teste $t$ de student para variáveis independentes.

De acordo com a Tabela 2, através da comparação antes e após o PCS obtidas pela aplicação do TCP, houve relevância significativa nas variáveis DP ( $\mathrm{p}=0,0117), \mathrm{FC}$ inicial $(p=0,0173)$ e Borg final $(p=0,0299)$.

Tabela 2 - Comparação das variáveis DP, FC inicial e final, Borg inicial e final e PAs e PAd inicial e final obtidas no TCP antes e após o PCS com resultados apresentados em médias e desvio padrão com os resultados do teste de Wilcoxon para variáveis dependentes.

\begin{tabular}{|c|c|c|c|}
\hline \multirow[b]{2}{*}{ Variáveis } & \multicolumn{2}{|c|}{ Programa de Caminhada Supervisionada } & \multirow{2}{*}{$\begin{array}{c}\text { Resultado } \\
\text { Estatístico } \\
\text { P }\end{array}$} \\
\hline & Avaliação & Reavaliação & \\
\hline DP & $180,25 \pm 30,94$ & $230,13 \pm 33,92$ & 0,0117 \\
\hline FC inicial & $80,13 \pm 10,97$ & $73,25 \pm 6,69$ & 0,0173 \\
\hline FC final & $116,63 \pm 29,24$ & $102,63 \pm 18,58$ & 0,1235 \\
\hline Borg Inicial & $0,00 \pm 0,00$ & $0,00 \pm 0,00$ & 1,0000 \\
\hline Borg Final & $3,38 \pm 1,41$ & $2,00 \pm 0,93$ & 0,0299 \\
\hline PAs Inicial & $135,00 \pm 13,09$ & $131,25 \pm 9,91$ & 0,1088 \\
\hline PAs Final & $147,50 \pm 20,53$ & $141,25 \pm 12,46$ & 0,3105 \\
\hline PAd Inicial & $81,25 \pm 8,35$ & $78,75 \pm 3,54$ & 0,3613 \\
\hline PAd Final & $90,00 \pm 11,95$ & $88,75 \pm 6,41$ & 1,0000 \\
\hline
\end{tabular}


A Tabela 3 evidencia alterações instrumento SF-36 e do escore Total do significativas para todos os domínios do questionário.

Tabela 3 - Comparação das variáveis dos domínios do questionário SF-36 antes e após o PCS com resultados apresentados em médias e desvio padrão com os resultados do teste de Wilcoxon para variáveis dependentes.

\begin{tabular}{lccc}
\hline \multicolumn{1}{c}{ Variáveis } & \multicolumn{2}{c}{$\begin{array}{c}\text { Programa de Caminhada } \\
\text { Supervisionada }\end{array}$} & $\begin{array}{c}\text { Resultado } \\
\text { Estatístico } \\
\text { Rvaliação }\end{array}$ \\
& Reavaliação & $85,63 \pm 9,43$ & 0,0117 \\
\hline Capacidade Funcional & $68,75 \pm 6,41$ & $87,50 \pm 13,36$ & 0,0117 \\
Limitação por Aspectos Físicos & $46,88 \pm 16,02$ & $86,00 \pm 13,73$ & 0,0179 \\
Dor & $65,75 \pm 17,65$ & $85,50 \pm 7,98$ & 0,0117 \\
Estado Geral de Saúde & $66,63 \pm 12,35$ & $74,38 \pm 10,84$ & 0,0117 \\
Vitalidade & $56,25 \pm 19,23$ & $86,13 \pm 10,47$ & 0,0179 \\
Aspectos Sociais & $64,13 \pm 12,38$ & $91,75 \pm 15,28$ & 0,0117 \\
Limitação por Aspectos Emocionais & $58,50 \pm 15,74$ & $76,50 \pm 16,34$ & 0,0117 \\
Saúde Mental & $60,50 \pm 19,88$ & $84,17 \pm 5,07$ & 0,0117 \\
Total & $60,92 \pm 7,95$ & \multicolumn{3}{c}{}
\end{tabular}

$\mathrm{Na}$ análise de correlação dos dados, entre as variáveis do TC6' e do TCP e o instrumento SF-36, segundo explanado na Tabela 4, houve correlação significativa entre a DP e o sub-domínio Capacidade Funcional $(p=0,0260)$ apenas quando comparado ao TCP, evidenciando que voluntários que apresentaram maior DP obtiveram maior escore neste domínio.

O presente estudo não apresentou, no entanto, correlação significativa entre 0 escore Total do questionário SF-36, bem como nos demais domínios, com as demais variáveis do TC6' e TCP, se analisados separadamente, tanto iniciais quanto finais, da FC, PAs, PAd e Borg.

Tabela 4 - Análise de Correlação entre a DP inicial e final do TC6' e do TCP e os escores dos domínios do Questionário SF-36.

\begin{tabular}{lcccc}
\hline \multicolumn{1}{c}{$\begin{array}{c}\text { Domínios } \\
\text { SF-36 }\end{array}$} & \multicolumn{2}{c}{ TC6' } & \multicolumn{2}{c}{ TCP } \\
Avaliação & Reavaliação & Avaliação & Reavaliação \\
\hline Capacidade Funcional & 0,4949 & 0,1498 & 0,6708 & 0,0260 \\
Limitação por Aspectos Físicos & 0,2663 & 0,5126 & 0,0593 & 0,7434 \\
Dor & 1,0000 & 0,7801 & 0,5049 & 0,7801 \\
Estado Geral de Saúde & 0,6075 & 0,7892 & 1,0000 & 0,7892 \\
Vitalidade & 0,6075 & 0,1814 & 0,1098 & 0,1814 \\
Aspectos Sociais & 0,6523 & 0,8864 & 0,2330 & 0,4750 \\
Limitação por Aspectos Emocionais & 0,0587 & 0,0587 & 0,8533 & 0,2568 \\
Saúde Mental & 0,7973 & 0,8649 & 0,4240 & 0,6075 \\
Total & 0,8997 & 0,6075 & 0,1503 & 0,8045 \\
\hline
\end{tabular}

$p \leq 0,05$. 'Significativo para teste $t$ de student para variáveis independentes.

\section{DISCUSSÃO}

Este estudo analisou as interações entre QV, SM e AF em 8 indivíduos, 7 do sexo feminino e 1 do sexo masculino, por meio de um PCS. O aumento cada vez mais crescente da longevidade e, consequentemente, da expectativa de vida, associados ao sedentarismo, tabagismo, dislipidemias, obesidade, predisposição 
genética, alimentação inadequada, HAS, DM2, favorece o aumento de riscos cardiovasculares e, portanto, da ocorrência de SM.

Estima-se que, em diferentes populações adultas, há uma elevada prevalência de indivíduos com SM, que varia entre $25 \%$ e $35 \%$, sendo mais frequente em mulheres, o que é condizente com nosso estudo, uma vez que voluntários apresentaram média de idade $63 \pm 9,65$ anos.

Cho et al. (2009) identificaram que a realização de atividades moderadas ou vigorosas é eficaz para a prevenção primária da SM, tanto para homens quanto para mulheres, independente da idade. No entanto, como observado em Pitsavos et al. (2006) pessoas sedentárias devem começar com exercícios de menor intensidade, especialmente indivíduos de meia-idade ou mais velhos, o que justifica a realização de caminhada como preditor da capacidade física e a utilização de testes de avaliação da capacidade máxima (TCP) e submáxima (TC6') de esforço.

A AF pode retardar e atenuar 0 declínio das funções orgânicas, com melhora da capacidade respiratória, reserva cardíaca, tempo de reação, força muscular, memória recente, cognição e habilidades sociais. Já Fappa et al. (2008), afirmam que a adesão ao tratamento do indivíduo com SM deve ser permanente para controle dos fatores de risco para SM.

Okura et al. (2007), em um programa de treinamento de 14 semanas, constataram que houve maior redução do tecido adiposo visceral em mulheres com sobrepeso e obesidade com a realização de exercícios físicos aeróbios juntamente à dieta para redução de peso do que apenas em programas com restrição dietética. Um estilo de vida, fisicamente ativo, com manutenção da aptidão cardiorrespiratória pode ser útil na prevenção primária da SM, com redução dos fatores de risco de doença cardíaca e coronariana.

Em outro estudo, Roussel et al. (2009) mostraram que um programa de caminhada com risco moderado, durante 16 semanas, há melhora do perfil metabólico de mulheres obesas sedentárias na pós-menopausa, com redução da gordura corporal e consequente melhoria do perfil de lipídios-lipoproteína. Os autores concluíram que o programa de caminhada é de grande importância para a promoção da saúde em mulheres moderadamente obesas entre 50 e 65 anos.

Warner et al. (2010), em um programa de treinamento de 8 a 12 semanas de exercício aeróbio em esteira com restrição dietética, seguido de um programa de exercício resistido de 8 a 12 semanas com controle de peso, identificaram redução em $6 \%$ os fatores de risco cardiometabólico e cardiorrespiratório. Os autores evidenciaram que as melhorias podem ser mantidas após a cessação do programa de treinamento e é um meio eficaz para manter e melhorar a aptidão cardiorrespiratória, força muscular e, portanto, poderia ser um componente vital para prescrição de exercícios para a redução do risco de SM e outras doenças crônicas.

Logo, o PCS proposto no presente estudo como forma de intervenção na SM é semelhante a um estudo de Pettman et al. (2008), que realizaram um programa de 
caminhada de 16 semanas, de forma intensiva, individualizada e acompanhado de alongamentos com monitoração da FC.

Sato et al. (2007) recomendam a realização de exercício aeróbio de intensidade ligeira a moderada, como caminhada, corrida, ginástica, bicicleta ergométrica e natação, de 10 a 30 minutos por dia, de três a cinco dias por semana. $O$ treinamento físico associado à dieta é eficaz na prevenção e tratamento de doenças insulino-dependente, como a SM, através da melhoria da RI. Segundo Okura et al. (2007), exercícios aeróbicos para redução do peso têm um efeito sinérgico na melhoria dos fatores de risco para SM.

O programa utilizado neste estudo com uma frequência de duas vezes por semana e de duração de 30 minutos/dia durante 10 semanas, pode-se verificar alterações significativas na DP, na sensação subjetiva de esforço e em outras variáveis, pós-treinamento que refletiram na melhora da QV dos voluntários.

De acordo com a DP, na comparação entre os testes aplicados, tanto no TC6' quanto no TCP, houve aumento dos escores na reavaliação dos voluntários após o término do PCS, o que identifica que o programa é eficaz para manter e melhorar a aptidão cardiorrespiratória, com redução da intolerância ao exercício e consequente redução do risco de SM e outras doenças crônicas, conforme exposto em estudos similares como em Okura et al. (2007), em um programa de treinamento de 14 semanas em indivíduos obesos e em Roussel et al. (2009), em um programa de treinamento de 16 semanas em mulheres obesas entre 50 e 65 anos.
Efeito similar pode ser observado com relação à sensação subjetiva de esforço por meio da escala de Borg modificada, uma vez que houve diminuição dos valores no momento da reavaliação do TC6' e do TCP em relação aos basais. Os participantes, portanto, apresentaram aumento da DP no final do PCS concomitantemente à diminuição de sensação de dispneia.

O presente estudo demonstra também, de acordo com a avaliação e reavaliação do TC6', que os voluntários apresentaram diminuição na variável FC ao final do teste, associado à DP, indicando que o PCS, foi efetivo em melhorar a capacidade cardiorrespiratória. Os voluntários caminharam mais no teste, com melhora na capacidade submáxima de exercício e menor sobrecarga circulatória, o que sugere que houve treinamento físico, semelhante ao exposto em Okura et al. (2007), em que evidenciam que um programa de caminhada promove diminuição do $\mathrm{VO}_{2} \max \mathrm{e}$ favorecem a diminuição da prevalência da $\mathrm{SM}$ e dos fatores de risco.

Outra variável que se modificou, sofrendo redução entre os momentos avaliação e reavaliação foi a $\mathrm{FC}$ de repouso, coletada antes do TCP, indicando que os voluntários apresentaram incremento da capacidade cardiovascular, com maior eficiência cardíaca em repouso. Os participantes apresentaram melhores índices basais da FC no início da reavaliação, evidenciando que estavam treinados, conforme avaliação da capacidade física máxima.

Com relação à avaliação da $\mathrm{PA}$ dos voluntários antes e após o PCS, o presente estudo mostrou que não houve alteração significativa dos valores da PAd e PAs, tanto 
no TC6' quanto no TCP, em relação aos valores basais no período de 10 semanas, que é condizente com um estudo de Balducci et al. (2009), que evidenciaram que, para a redução de níveis pressóricos, são necessários protocolos de treinamento de no mínimo três semanas até 12 meses, associado ao controle alimentar. A utilização do TC6' e do TCP, por meio de suas variáveis, como forma de avaliação da capacidade cardiorrespiratória, é eficaz para identificação da efetividade de um treinamento. Em ambos os testes a DP pelo indivíduo é a variável mais importante e que permite avaliar o desempenho físico, permitindo a análise de correlação com a pontuação do questionário de QV.

A aplicação do questionário de QV SF-36 evidencia que pessoas com SM apresentam pior índice que os que não possuem a síndrome, e que interferem de forma negativa na QV, como em um estudo de Tsai et al. (2007), em que indivíduos com SM apresentaram menor pontuação no questionário SF-36, além de sintomas de depressão, uso de álcool ou tabaco e estresse.

Han et al. (2009), demonstraram que indivíduos com SM possuem uma QV significativa menor em comparação com as sem a doença. Pessoas com SM são mais propensas a apresentar problemas com a saúde física, saúde relacionada ao trabalho, vida cotidiana, vida sexual, angústia com a dieta e dor ou desconforto.

O presente estudo, por meio da análise da interação entre $Q V$ e $A F$, evidenciou que todos os voluntários obtiveram melhora da capacidade física por meio do TC6' e do TCP, com aumento da DP após o período de treinamento com diminuição da sensação subjetiva de esforço o que influiu tanto no escore total do questionário SF-36 quanto em seus domínios se analisados separadamente.

Conforme exposto por Silva e Nahas (2002), o treinamento aumenta a capacidade de caminhar, com mudanças significativas da distância, velocidade, tempo de caminhada, melhora do fluxo sanguíneo periférico, melhora do metabolismo musculoesquelético, facilitando a utilização do oxigênio. Todas essas alterações oriundas da pratica de AF reduzem os sintomas da SM e melhoram a $Q V$ do indivíduo, percepção do bem-estar e disposição.

Devido ao PCS e os benefícios da AF e sua vinculação à aplicação do questionário SF-36, pode-se afirmar que houve aumento, com relevância significativa, de todos os escores do questionário após o programa: capacidade funcional, limitação por aspectos físicos, dor, estado geral de saúde, vitalidade, aspectos sociais, limitação por aspectos emocionais, saúde mental e total.

A realização de exercícios físicos promove melhora nos domínios do questionário SF-36 em relação a indivíduos que não realizam $A F$, seja por meio de programas de caminhada, treinamento de força muscular, resistência, flexibilidade ou alongamento. $\mathrm{O}$ mesmo pode ser observado em Brito et al. (2008), que evidenciaram que a AF regular melhora os domínios capacidade funcional, estado geral de saúde, vitalidade, aspectos sociais e saúde mental do SF-36.

$\mathrm{Na}$ análise de correlações entre as 
variáveis DP nos testes aplicados e os escores do SF-36 foi encontrada relevância significativa na comparação da DP do TCP com o domínio capacidade funcional do questionário SF-36 e evidencia que foi eficaz para a melhora da DP dos voluntários. Os indivíduos que caminharam mais durante a reavaliação do TCP apresentaram melhor escore nesse domínio, o que é semelhante ao objeto deste trabalho: pessoas que realizam AF apresentam aumento da capacidade de caminhar e de realizar as atividades de vida diária.

Em um estudo norte-americano, Sullivan et al. (2007) evidenciaram que indivíduos com alterações cardiometabólicas como sobrepeso, obesidade, DM2, HAS e hiperlipidemia apresentaram um impacto significativo e negativo nas QV na população dos Estados Unidos.

Tsai et al. (2007) estudaram a associação entre SM e QV e constataram que houve menor pontuação na função física e sub-escalas de saúde geral do questionário SF-36. Indivíduos com SM relataram menor QV, sintomas de depressão, uso de álcool ou tabaco e estresse, bem como um significativo maior IMC do que aqueles sem a síndrome.

Rush, Chandu e Plank (2007) avaliaram o impacto de uma dieta e AF em grupo de idosos asiáticos sobre a composição corporal, perfil lipídico no sangue e RI. Foram realizadas reuniões mensais de educação em grupo sobre dieta, AF e da importância das mudanças de estilo de vida para redução dos fatores de risco para doença crônica e concluíram que, após um período de cinco meses, houve redução significativa no total de gordura corporal e abdominal e dos fatores de risco.
Em outro estudo, Pinotti, Mantovani e Giacomozzi (2008) avaliaram a QV em indivíduos hipertensos e verificaram que a HAS interferiu de maneira negativa, principalmente em relação ao trabalho, hábitos de vida e autocuidado decorrente do caráter crônico da doença.

Os demais domínios do SF-36 não apresentaram correlação significativa entre as variáveis do TC6' e do TCP e entre as hipóteses para tal estão: a) amostra constituída por um pequeno número de voluntários; ou b) o presente estudo não verificou a influência direta no dia a dia dos voluntários, por meio de intervenção educacional, controle nutricional ou mudanças no estilo de vida.

Pitsavos et al. (2008) ressaltam a modificação dos hábitos de vida como fator associado à $\mathrm{AF}$ para melhora da QV em pessoas com SM e redução dos fatores de risco cardiovascular, como sedentarismo e controle do aporte nutricional.

Outro ponto não analisado $e$ correlacionado no presente estudo refere-se à mensuração invasiva de alterações metabólicas da SM, tais como glicemia, TG, HDL-colesterol, controle de peso, medida da circunferência abdominal e controle alimentar antes e depois do PCS. Estes valores, além de critérios para determinação da SM, poderiam ser utilizados como escores comparativos da evolução metabólica do indivíduo pós AF com relação a RI e níveis lipídicos, e não apenas variáveis relacionadas ao desempenho físico.

Mudanças no estilo de vida (dieta e exercício físico) constituem um importante componente para controle da SM. A maior 
adesão a essas mudanças promovem melhoras nos fatores prejudiciais da doença. Além disso, as modificações não devem ser provisórias, mas sim permanentes e adesão permanente pelo portador deve ser considerada como um componente essencial.

\section{CONCLUSÃO}

De acordo com os resultados obtidos do presente estudo, podemos concluir que a realização de $\mathrm{AF}$ regular é eficaz para o controle dos sintomas relacionados à SM. A obesidade, o sedentarismo, a HAS, a DM2, dislipidemia, altos níveis de LDL e TG favorecem 0 aumento dos riscos cardiovasculares e estes precisam ser controlados para diminuição da morbidade e mortalidade principalmente em indivíduos idosos.

O programa mostrou-se satisfatório para incremento dos escores de todos os domínios do questionário SF-36, o que evidencia que a aplicação do instrumento foi adequada para a avaliação da QV na amostra estudada. Com isso, pode-se ratificar a importância do condicionamento físico para melhora do bem-estar, da socialização da auto-estima, e principalmente da QV.

\section{REFERÊNCIAS}

BALDUCCI, S. et al. Anti-inflammatory effect of exercise training in subjects with type 2 diabetes and the metabolic syndrome is dependent on exercise modalities and independent of weight loss. Nutr. Metab.

Cardiovasc. Dis., v. 10, 2009.
BARBOSA, J. B. et al. Síndrome metabólica em ambulatório cardiológico. Arq. Bras. Cardiol., v. 94, n. 1, p. 46-54, 2010.

BRITO, D. M. S. et al. Qualidade de vida e percepção da doença entre portadores de hipertensão arterial. Cad. Saúde Pública, Rio de Janeiro, v. 24, n. 4, p. 933-940, abr. 2008.

CAMPOS, F. V. de S.; PORTO, L. G. G. Qualidade de vida e nível de atividade física de pacientes em fase ambulatorial da reabilitação cardíaca. Revista Brasileira de Atividade Física \& Saúde, Brasília, v. 14, n. 2, p. 86-95, mai.-ago. 2009.

CARVALHO, E. M. G.; RABELO, J. N. R. Identificação, prevenção e tratamento dos fatores de riscos associados à síndrome metabólica em pacientes atendidos no programa integrado de atividade física, esporte e lazer para todos os servidores da UFV campus florestal: estudo piloto - PiafelEP. Synthesis Revista Digital FAPAM, Pará de Minas, n. 1, 2009.

CAVALCANTE, M. A. et al. Qualidade de vida de pacientes hipertensos em tratamento ambulatorial. Arq. Bras. Cardiol., São Paulo, v. 89, n. 4, p. 245-250, out. 2007.

$\mathrm{CHO}$, E. R. et al. Leisure-time physical activity is associated with a reduced risk for metabolic syndrome. AEP, v. 19, n. 11, p. 784-792, nov. 2009.

COELHO, C. C. et al. Análise comparativa e reprodutibilidade do teste de caminhada com carga progressiva (modificado) em crianças normais e em portadoras de fibrose cística. J. Bras. Pneumol., v. 33, n. 2, p. 168-174, 2007.

ELIAS, R. G. M. et al. Influência da atividade 
física sobre a prevalência de Síndrome metabólica, em mulheres atendidas em uma unidade básica de saúde, Maringá - PR. Cienc. Cuid. Saude, v. 7, suplem. 1, p. 8893, 2008.

FAPPA, E. et al. Lifestyle intervention in the management of metabolic syndrome: could we improve adherence issues? Nutrition, $v$. 24, p. 286-291, 2008.

GELONESE, B. Síndrome metabólica: mito ou realidade? Arq. Bras. Endocrinol. Metab., v. 50, n. 3, p. 409-411, jun. 2006.

HAN, J. H. et al. Metabolic syndrome and quality of life (QOL) using generalised and obesity-specific QOL scales. Int. J. Clin. Pract., v. 63, n. 5, p. 735-741, mai. 2009.

\section{DIRETRIZ BRASILEIRA DE DIAGNÓSTICO E TRATAMENTO DA SÍNDROME METABÓLICA. Arquivos Brasileiros de Cardiologia, v.84, suplemento I, p. 1-28, abr. 2005.}

JUNQUEIRA, A. S. M.; ROMÊO FILHO, L. J. M.; JUNQUEIRA, C. de L. C. Avaliação do grau de inflamação vascular em pacientes com síndrome metabólica. Arq. Bras. Cardiol., v. 93, n. 3., p. 360-366, 2009.

LOTTENBERG, S. A.; GLEZER, A.; TURATTI, L. A. Síndrome metabólica: identificando fatores de risco. Jornal de Pediatria, Rio de Janeiro, v. 83, suplemento 5, p. S204-S208, 2007.

LUNA, R. L. Síndrome metabólica. Arq. Bras. Cardiol., v. 88, n. 5, p. e124-e126, 2007.

MANNA, T. D.; DAMIANI, D.; SETIAN, N. Síndrome metabólica: revisão. Pediatria, São Paulo, v. 28, n. 4, p. 272-277, 2006.

McLELLAN, K. C. P. et al. Diabetes mellitus do tipo 2, síndrome metabólica e modificação no estilo de vida. Revista Nutrição, Campinas, v. 20, n. 5, p. 515-524, set.-out. 2007

MIRANZI, S. de S. C. et al. Qualidade de vida de indivíduos com diabetes mellitus e hipertensão acompanhados por uma equipe de saúde da família. Texto Contexto Enferm., Florianópolis, v. 17, n. 4, p. 672 679, out.-dez. 2008.

MOTA, J. et al. Atividade física e qualidade de vida associada à saúde em idosos participantes e não participantes em programas regulares de atividade física. Rev. Bras. Educ. Fís. Esp., São Paulo, v. 20, n. 3, p. 219-225, jul.-set. 2006.

NAKAZONE, M. A. et al. Prevalência de síndrome metabólica em indivíduos brasileiros pelos critérios de NCEP-ATPIII e IDF. Rev. Assoc. Med. Bras., v. 53, n. 5, p. 407-13, 2007.

OKURA; T. et al. Effects of aerobic exercise on metabolic syndrome improvement in response to weight reduction. Obesity., v. 15, n. 10, p. 2478-2484, out. 2007.

PETTMAN, T. L. et al. Self-management for obesity and cardio-metabolic fitness: Description and evaluation of the lifestyle modification program of a randomised controlled Trial. International Journal of Behavioral Nutrition and Physical Activity, v. 5, n. 1, p. 53-67, out. 2008.

PINOTTI, S.; MANTOVANI, M. de F.; GIACOMOZZI, L. M. Percepção sobre a hipertensão arterial e qualidade de vida: contribuição para o cuidado de enfermagem. Cogitare enferm., v. 13 , n. 4 , p. 526-534, out.-dez. 2008.

PITSAVOS, C. et al. Diet, Exercise and the 
Metabolic Syndrome. Rev. Diabetic Stud, v. 3, n. 3, p. 118-126, 2006.

PUGLIESE, et al. Self glucose monitoring and physical exercise in diabetes. Diabetes Metab. Res. Rev., v. 25, Suppl. 1, p. S11S17, 2009.

RIGO, J. C. et al. Prevalência de síndrome metabólica em idosos de uma comunidade: comparação entre três métodos diagnósticos. Arq. Bras. Cardiol., v. 93, n. 2, p. 85-91, 2009.

ROUSSEL, M. et al. Influence of a walking program on the metabolic risk profile of obese postmenopausal women. Menopause, v. 16, n. 3, p. 566-575, 2009.

RUSH, E. C.; CHANDU, V.; PLANK, L. D. Reduction of abdominal fat and chronic disease factors by lifestyle change in migrant Asian Indians older than 50 years. Asia Pac. J. Clin. Nutr., v. 16, n. 4, p. 671-676, mai. 2007.

SATO, Y. et al. Clinical aspects of physical exercise for diabetes / metabolic syndrome.

Diabetes Research and Clinical Practice, v. 77S, p. S87-S91, 2007.

SILVA, D. K. da; NAHAS, M. V. Prescrição de exercícios físicos para pessoas com doença vascular periférica. Rev. Bras. Ciên. e Mov.,
Brasília, v. 10, n. 1, p. 55-61, jan. 2002.

SILVA, D. A. S.; JESUS, K. P. de; SANTOS, R. J. dos. Conceito de saúde e qualidade de vida para acadêmicos de educação física um estudo descritivo. Revista Brasileira de Educação Física, Esporte, Lazer e Dança, v. 2, n. 4, p. 140-153, dez. 2007.

SULLIVAN, P. W. Impact of cardiometabolic risk factor clusters on health-related quality of life in the U.S. Obesity, v. 15, n. 2, p. 511521, fev. 2007.

TSAI, A. G. et al. Metabolic syndrome and health-related quality of life in obese individuals seeking weight reduction. Obesity, v. 16, n. 1, p. 59-63, jan. 2007.

WARNER, S. O. et al. The effects of resistance training on metabolic health with weight regain. The journal of clinical hypertension, v. 12 , n. 1, p. 64-72, jan. 2010.

WILUND, K. R. et al. Effects of endurance exercise training on markers of cholesterol absorption and synthesis. Physiol. Res., v. 58, p. 545-552, 2009.

WINKELMANN, E. R.; MANFROI, W. C. Qualidade de vida em cardiologia. Rev. HCPA, Porto Alegre, v. 28, n. 1, p. 49-53, 2008. 